\title{
Un paradigme totalement différent
}

Jean Martin

Dr med., membre de la rédaction

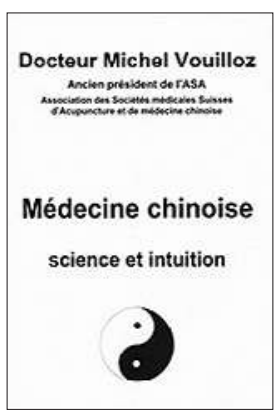

Michel Vouilloz Médecine chinoise Science et intuition

\section{Sembrancher:} Imprimerie des 3 Dranses; 2015 104 pages. $20 \mathrm{CHF}$. ISBN 978-2-839-91773-5 Mis en vente par SATAS, Editeur Chaussée de Ninove, 1072,

1080 Saint-Jans-

Molenbeek, Belgique Tél: +32 25696989
Le Dr Michel Vouilloz, spécialiste FMH en médecine interne, a montré au cours de sa carrière la diversité de ses intérêts et engagements en étant, entre autres choses, médecin-chef de la Croix-Rouge suisse, puis médecin cantonal du Valais. Intéressé par la médecine chinoise depuis ses études, il a consacré les dernières décennies de sa carrière à la pratique de l'acupuncture à Martigny.

Après un Dictionnaire de médecine chinoise à l'usage des profanes curieux en 2008, il publie Médecine chinoise, en y rassemblant ce qu'il a appris et pratiqué quant à la médecine traditionnelle de la Chine. Ce qui interpelle, c'est que, dans le cadre d'un effort substantiel historique, descriptif, comparatif et épistémologique, il ne cherche en rien à dire que cette médecine devrait retenir notre attention parce, après tout, elle pourrait être comparée à la nôtre. Au contraire, il explique comment il n'est guère possible de les considérer en parallèle (même si des travaux scientifiques ont validé les effets de l'acupuncture par exemple), parce qu'elles procèdent de fondements complètement différents.

Le Dr H. Dong, qui préface l'ouvrage: «Le Taoïsme [d'où découle la médecine chinoise] s'intéressa volontairement plus à la sagesse intuitive qu'à la connaissance rationnelle. Néanmoins, l'observation attentive de la nature combinée à une forte perspicacité mystique amena les sages à des intuitions qui furent confirmées par les découvertes de physique et de médecine modernes». Médecine occidentale rationnelle, médecine chinoise de l'intuition... Dong: "Aucune des deux n'est incluse dans l'autre ni ne peut être réduite à l'autre, mais elles sont nécessaires et l'homme a besoin des deux".

A discuter. C'est ce qu'entend faire notre confrère Vouilloz: «La conception chinoise de la vie, et de l'Homme, holistique, procède par une approche globale, jamais par analyse, jugée trop réductionniste. Elle ne s'est jamais intéressée à l'anatomie. Elle estime que la recherche d'une étiologie est inadéquate étant donné la complexité de la vie.» «La culture occidentale voit des pathologies greffées sur un être normal ce qui est aux antipodes de la culture chinoise. En fait, l'anomalie est consubstantielle à l'être humain. La méde- cine chinoise s'intéresse à la dysharmonie, la question qu'elle pose est: qu'est-ce qu'il faudrait équilibrer?» On est là devant une forme d'incommunicabilité entre «eux» et «nous». Les bases mêmes d'un dialogue, au plan théorique pour le moins, ne semblent pas réunies. Pourtant, d'une part il faut toujours dialoguer. D'autre part, des modalités thérapeutiques chinoises, l'acupuncture en particulier, sont maintenant admises dans nos pays et facultés. La réalité demande, volens nolens, de reconnaître des faits alors même qu'on ne sait pas en intégrer les fondements théoriques (ou l'absence de tels fondements!) dans nos systèmes de référence. Difficile pour ceux de ma génération, éduqués dans un cadre sûr de lui n'admettant que le rationnel. Apprendre à vivre avec ces éléments pas explicables dans notre rationalité mais qu'on ne peut plus traiter de pures illusions. Qui sont du registre de l'épi-, du para-, du «soft», du non-rectiligne, de l'empirique/ pragmatique, du qualitatif. Un monde intellectuellement nettement moins confortable.

Dans sa conclusion, Michel Vouilloz relève que le dialogue entre Chine et Occident qui souhaiterait se baser sur la médecine factuelle (basées sur les preuves) n'a pas donné de résultats bien convaincants jusqu'ici. «Outre ce bilan mitigé», dit-il, "ce livre constate la grande modernité des concepts chinois, avec un mélange d'intuition et de raisonnement YinYang. La vision mécaniste européenne a obtenu d'indéniables succès, mais aujourd'hui la physique a quitté les certitudes pour des probabilités, où s'insinue dans l'univers l'existence de l'énergie du vide.»

Ces interrogations sont posées, les limites vite atteintes de mes compétences m'interdisent de conclure. Médecine chinoise n'est pas un traité sophistiqué, mais l'essai informé d'un praticien qui a vécu le potentiel, les réussites et sans doute aussi les échecs des deux médecines. Praticien qui cherche à faire œuvre de pont, de passeur - sous une forme ramassée et facile à lire. On peut lui en savoir gré, pas seulement au plan d'une réflexion sur les arts de soigner mais plus généralement pour l'ouverture proposée à un système de pensée, et de «fonctionnement», si différent du nôtre. 